TTR

Traduction, terminologie, rédaction

\title{
« These foreign c*nts've goat trouble wi the Queen's f*ckin English, ken ", ou ré-énoncer la voix scots de Trainspotting au moyen du Québec et de la (socio-)linguistique
}

\section{Alex Gauthier}

Volume 28, numéro 1-2, 1er semestre-2e semestre 2015

La traduction littéraire et le Canada

Literary translation and Canada

URI : https://id.erudit.org/iderudit/1041657ar

DOI : https://doi.org/10.7202/1041657ar

Aller au sommaire du numéro

Éditeur(s)

Association canadienne de traductologie

ISSN

0835-8443 (imprimé)

1708-2188 (numérique)

Découvrir la revue

Citer cet article

Gauthier, A. (2015). « These foreign c*nts've goat trouble wi the Queen's f*ckin English, ken ", ou ré-énoncer la voix scots de Trainspotting au moyen du Québec et de la (socio-)linguistique. TTR, 28(1-2), 207-237.

https://doi.org/10.7202/1041657ar
Résumé de l'article

En 1993, au lendemain du règne thatchérien, Irvine Welsh, auteur écossais alors inconnu, était propulsé au firmament des vedettes littéraires grâce à Trainspotting, un texte coup de poing mettant en scène de jeunes héroïnomanes écossais qui s'opposent au système de la seule manière qu'ils le peuvent : à coup d'injections. En les faisant s'exprimer dans une version extrêmement codée du scots, Welsh déployait un système discursif unique et volontairement difficile à déchiffrer, véritable doigt d'honneur à l'élite littéraire anglophone et aux conventions de l'écriture. Dans cet article, j'aborde premièrement l'importance du sociolecte scots dans l'oeuvre de Welsh, au moyen d'une analyse du contexte sociohistorique de l'oeuvre et de sa réception. Deuxièmement et à contresens des traducteurs français (Lindor Fall en 1996, Étienne en 2011), qui livrent des versions qui répriment l'insubordination de l'original aux normes de la langue, je présente une " systématique de la déformation " basée sur des études sociolinguistiques du français québécois, qui permet de ré-énoncer, pour le lecteur francophone, la puissance expressive et la portée politique de la voix de ce texte culte. Par l'utilisation d'une variante du français québécois vernaculaire comme outil de traduction, j'espère ménager dans l'espace littéraire francophone une ouverture qui permette d'éviter la notion d'intraduisible lorsque l'on parle des sociolectes. 


\title{
"These foreign $\mathrm{c}^{*}$ nts've goat trouble wi the Queen's f*kin English, ken ", ou ré-énoncer la voix scots de Trainspotting au moyen du Québec et de la (socio-)linguistique ${ }^{1}$
}

\author{
Alex Gauthier \\ Université Concordia
}

\section{Résumé}

En 1993, au lendemain du règne thatchérien, Irvine Welsh, auteur écossais alors inconnu, était propulsé au firmament des vedettes littéraires grâce à Trainspotting, un texte coup de poing mettant en scène de jeunes héroïnomanes écossais qui s'opposent au système de la seule manière qu'ils le peuvent: à coup d'injections. En les faisant s'exprimer dans une version extrêmement codée du scots, Welsh déployait un système discursif unique et volontairement difficile à déchiffrer, véritable doigt d'honneur à l'élite littéraire anglophone et aux conventions de l'écriture. Dans cet article, j'aborde premièrement l'importance du sociolecte scots dans l'ouvre de Welsh, au moyen d'une analyse du contexte sociohistorique de l'œuvre et de sa réception. Deuxièmement et à contresens des traducteurs français (Lindor Fall en 1996, Étienne en 2011), qui livrent des versions qui répriment l'insubordination de loriginal aux normes de la langue, je présente une «systématique de la déformation» basée sur des études sociolinguistiques du français québécois, qui permet de ré-énoncer, pour le lecteur francophone, la puissance expressive et la portée politique de la voix de ce texte culte. Par l'utilisation d'une variante du français québécois vernaculaire comme outil de traduction, j’espère ménager dans l'espace littéraire francophone une ouverture qui permette d'éviter la notion d'intraduisible lorsque l'on parle des sociolectes.

Mots-clés: Henri Meschonnic, sociolecte, sociolinguistique, Irvine Welsh, Trainspotting

\section{Abstract}

In 1993, in the aftermath of the Thatcherian era, Irvine Welsh, then an unknown Scottish writer, was propelled in the high spheres of the literary scene, thanks to Trainspotting, a hard-hitting novel featuring drug-addled Scottish twenty-somethings that oppose the system the only way they can:

1. Cet article reprend des passages de mon mémoire de maitrise, accessible au http://spectrum.library.concordia.ca/982297/. 
through injections of heroin. By making them talk in an extremely cryptic version of Scots, Welsh put forth a unique discursive system that mocks both the English-speaking literary elites and the writing conventions of English. In this article, I examine the significance of the Scots sociolect in Welsh's novel through an analysis of its socio-historical context and reception, and show how the novel's French translators (Lindor Fall in 1996 and Étienne in 2011) produced versions that suppress the insubordination of the source text's language. I then present a "system of textual deformation" anchored in Québécois sociolinguistic studies, that reproduces, for the French-speaking reader, the political significance and expressive strength of this cult novel's Meschonnician voice. By using a variation of Quebec's vernacular French as a translation tool, I strive to create a space, in the francophone literary landscape, where sociolects are no longer considered untranslatable.

Keywords: Henri Meschonnic, sociolects, sociolinguistics, Irvine Welsh, Trainspotting

En 1993, Irvine Welsh chamboulait le monde littéraire anglophone avec Trainspotting, un roman devenu culte mettant en scène à Édimbourg de jeunes hérö̈nomanes révoltés, vivotant dans une Écosse aliénée par le néolibéralisme de Thatcher. Plus que les personnages irrévérencieux, c'est la langue, outrageuse et profondément marginale dans son orthographe, qui bouscule le lecteur. Welsh, ainsi, force le lecteur à décoder le texte pour comprendre ses personnages, dont l'identité même repose sur leur utilisation du sociolecte ${ }^{2}$ scots et sur le rapport qu'ils entretiennent avec celui-ci et la langue anglaise standard. Le scots est également au cœur du message du roman, et son épaisseur graphique peut être vue comme une résistance et une insoumission à l'anglais, un doigt d'honneur lancé à l'Angleterre qui, dans les années 1980 et 1990, a abandonné l'Écosse de quadruple façon: économiquement, politiquement, culturellement et linguistiquement.

Les représentations sociolectales participent en effet «d'une stratégie latente de contestation non seulement de l'hégémonie du bien écrire, mais aussi des rapports de place et de force, des savoirs

2. J'utilise le terme sociolecte tel que défini par Annick Chapdelaine et Gillian Lane-Mercier, soit un «terme générique qui recouvre ceux, plus spécifiques car fondés sur un ensemble plus restreint de paramètres, de vernaculaire, qui désigne le parler d'un groupe ethnique en marge de la langue officielle comme des instances de pouvoir, de patois, qui renvoie au seul parler paysan, de pidgin et de créole, basés surtout sur des critères de formation linguistique et d'appartenance ethno-géographique, de dialecte, enfin, où les déterminations géographiques impliquent en règle générale des déterminations socioculturelles» (1994, p. 8). 
et des croyances valorisés et mis en circulation par les discours "bien pensants" de l'idéologie dominante» (Lane-Mercier, 1995, p. 81). Le sociolecte, par sa nature souvent politique, pose ainsi un épineux problème, celui de comment le traduire. Antoine Berman lui-même affirmait en 1985 que l'on ne peut traduire un sociolecte par un autre sociolecte (p.79), un mot d'ordre assez répandu pour qu'Annick Chapdelaine parle d'une «tradition française de non-représentation littéraire des sociolectes»(1996, p. 94). Plus récemment, dans Éthique et politique du traduire, Henri Meschonnic parlait de la résignation du traducteur, qui ne traduit que le sens dans les textes littéraires:

$[\mathrm{O}] \mathrm{n}$ ne traduit que du signe. Le poème est effacé. Mais tout le monde sait que le poème est effacé. C'est même parce qu'on le sait qu'on dit qu'il y a de l'intraduisible. On se résigne. On est habitué à se résigner. Le traducteur du signe est une âme habituée. [...] Traduire le signe, [...] c'est ne pas avoir de voix. Le signe rend aphone, en même temps qu'il rend sourd. (2007, p. 151)

Cependant, dans le cas d'un texte politisé comme Trainspotting, le traducteur ne peut pas «se résigner» lorsque vient le temps de traduire. Il doit infiltrer le système linguistique, en maîtriser les moyens et ensuite déployer un système d'expression cohérent parce que systématique. Meschonnic préférait le verbe traduire au nom traduction justement parce qu'il implique une action et une intention, et parce qu'il doit être fait par quelqu'un. Traduire, donc, veut dire agir sur le texte et déformer certes, mais aussi et surtout chercher à rendre ce qu'il fait: contester l'ordre établi.

Dans cet article, je propose premièrement d'aborder l'importance du sociolecte scots dans l'œuvre de Welsh, au moyen d'une analyse du contexte sociohistorique de l'œuvre et de sa réception. Deuxièmement, et à contresens des traducteurs français (Lindor Fall en 1996, Étienne en 2011), qui livrent des versions qui répriment l'insubordination de l'original aux normes de la langue, je présente une "systématique de la déformation» basée sur des études sociolinguistiques du français québécois, qui permet de ré-énoncer, pour le lecteur francophone, la puissance expressive et la portée politique de la voix de ce texte culte. Par l'utilisation d'une variante du sociolecte québécois comme outil de traduction, j'espère donc ménager dans l'espace littéraire francophone une ouverture qui permette d'éviter la notion d'intraduisible lorsque l'on parle des sociolectes. 


\section{Le scots comme vecteur de l'incompréhension}

«Whair's it yis come fae then?» C'est la question que pose Begbie, un des personnages écossais de Trainspotting, à deux Canadiennes dans un train se rendant à Londres. La question - d'où venez-vous? - trahit dès son élocution l'origine de celui qui la pose. Elle marque, géographiquement, socialement, son auteur. Dans la scène, tirée du chapitre "Intershitty», la question a aussi un effet indésirable. Cet appel à l'autre, en raison de sa forme, rate sa cible.

«Sorry, I can't really understand you», répond une des Canadiennes, troublée. Cette incompréhension, cette incapacité même à communiquer, est présente tout au long du roman Trainspotting de Irvine Welsh. Elle est thème, évidemment, mais aussi texte. Elle est la voix, au sens meschonnicien, du roman. Bien sûr, on a ici affaire à un dialogue. Toutefois, cette langue, représentation de l'accent scots, est présente aussi dans les phases narratives, et la narration en anglais dit standard est en fait si ponctuelle qu'elle détonne. C'est elle qui devient étrangère dans le roman. Le constat est clair: Trainspotting se veut difficile à lire. Lors d'une entrevue qu'il accorde à Andréa Burzinsky pour Reuters en 2012, Welsh évalue qu'il faut au moins trente pages au lecteur pour déchiffrer adéquatement la langue et atteindre un rythme de lecture normal. Welsh, d'ailleurs, refusait mordicus que son roman contienne un glossaire. Pas question d'aider le lecteur, surtout pas l'élite, que Welsh appelle gentiment those fuckers, et qui, selon lui, n'en retirerait que quelques moqueries supplémentaires à lancer aux Écossais dans un accent parodique. Non: pour lire Trainspotting, le lecteur doit apprivoiser une langue et un univers de gens qui, selon l'écrivain Andrew O'Hagan, sont habituellement ignorés dans la littérature (cité dans McKay, 1996, n.p.). Pour lire Trainspotting, le lecteur doit travailler. L'Histoire nous apprend que le lectorat anglophone l'a fait volontiers.

\section{Réception et effet}

Trainspotting est un roman-culte assorti d'une adaptation cinématographique tout aussi idolâtrée réalisée par Danny Boyle en 1996. Publié en 1993 aux éditions Martin Secker and Warburg, il est d'abord tiré à 3000 exemplaires. Il s'en vendra plus de 150000 durant ses trois premières années d'existence, et le roman sera réimprimé seize fois. À partir de 1996, sans doute motivées par l'engouement entourant l'adaptation cinématographique de Danny Boyle, une multitude de maisons d'édition publient des traductions 
qui seront célébrées partout dans le monde, et ce, dans plus de onze langues. En 2006, ce nombre passe à vingt-cinq.

Son auteur, Irvine Welsh, devient du jour au lendemain l'incontournable mauvais garçon de la littérature de langue anglaise, et la presse en fait ses choux gras en le décrivant de mille et une façons: "l'homme sauvage de la littérature, le plus essentiel des auteurs contemporains, le leader d'une renaissance culturelle, le créateur du "British Buzz", le sauveur de la littérature britannique, un monument national, le poète lauréat de la "chemical generation” (Morace, 2001, p. 7; ma trad.). Le roman, pour sa part, reçoit autant de bonnes critiques que de mauvaises. Ses détracteurs l'accusent de glorifier la toxicomanie à des fins sensationnalistes et de donner trop de détails sur la manière d'utiliser l'héroïne, et l'on craint qu'il ne serve de mode d'emploi pour les jeunes en quête de sensations fortes. (L'influence de Trainspotting sur ce plan reste encore à prouver.) Une critique plus justifiée vise à la fois Welsh et Danny Boyle, le réalisateur de l'adaptation cinématographique: on rend l'héroïne cool. Les personnages sont attirants dans leurs imperfections, leur dégaine et leur humour noir, et, dans le cas du film, ils sont mis en scène de manière hyper léchée, au son de la musique du moment.

Dans les sphères underground, les critiques sont dithyrambiques. Le magazine écossais alternatif Rebel, Inc. va jusqu'à décrire Trainspotting comme «le meilleur livre jamais écrit... il mérite de vendre plus d'exemplaires que la Bible» (Crawford, 2009, p. 672; ma trad.). Trainspotting est pressenti pour le prestigieux Man Booker Prize, qui lui échappera toutefois après avoir soi-disant choqué les valeurs de certains juges. En 1993, le roman est sacré meilleur livre de fiction par The Guardian. Selon Robert Morace, Welsh est devenu dans les années 1990 et 2000 le point de comparaison de la littérature britannique: «New writers were greeted and graded according to the Welsh standard. They were as good as Welsh or not as good; [...] the next Welsh, the female Welsh, the English Welsh, the Welsh Welsh, the Muslim Welsh» (2007, p. 13).

\section{Trainspotting, représentation du tourment identitaire de l'Écosse}

Or, si le roman soulève tant les passions, c'est qu'il arrive à une période extrêmement tendue au Royaume-Uni: en pleine ère thatchérienne. L'identité britannique est fracturée et celle des Écossais, encore plus. Après l'échec en 1979 d'un référendum de régionalisation des 
pouvoirs qui aurait donné une certaine indépendance à l'Écosse, Margaret Thatcher instaure des politiques qui visent à rapatrier le pouvoir et l'économie à Londres. L'emploi public, dont l'Écosse dépend grandement, est saigné à blanc, et l'aide financière qu'on accordait à l'Écosse depuis 1945 est coupée. Thatcher veut faire du Royaume-Uni une société post-industrielle et baser son économie sur le secteur tertiaire, une visée qui entre en conflit direct avec les principaux secteurs industriels écossais, la sidérurgie et la construction navale. En pleine crise économique, l'Écosse se retrouve donc caduque, avec un taux de chômage frisant les $13 \%$ et, si l'on en croit les sondages de l'époque, une haine profonde à l'endroit de Margaret Thatcher (Mitchell et Bennie, 1996, p. 96).

Culturellement, le pouvoir de la classe ouvrière est aussi amoindri par l'introduction du libre marché et le besoin décroissant de cols bleus. La culture change: ses produits sont manufacturés à la chaîne par l'industrie de la culture, qui «appose désormais la même étampe sur tout. Les films, la radio et les magazines forment un système en tout et pour tout uniforme" (Adorno et Horkheimer, 2002 [1944], p. 94; ma trad.). La culture et la société britanniques se tournent vers l'international, évacuant par la même occasion tout leur symbolisme local. La langue écossaise écope de ce lissage culturel, parce qu'il ne permet plus de présenter dans les produits de culture des personnages issus de la classe ouvrière écossaise. L'identité et la langue scots deviennent ainsi des symboles de honte, de capitulation. Welsh disait d'ailleurs, en parlant de l'époque, qu' ' [i]1 y a toujours eu cette idée qu'il existe deux sortes d'Écossais: celui qui va à Londres et qui gagne le gros lot, et l'autre, de second ordre, qui reste au bercail»(Edemariam et Scott, 2009; ma trad.). L'Écosse subit donc un quadruple abandon: politique, économique, culturel et linguistique. Dans Trainspotting, les Écossais traînent le lourd fardeau de la défaite politique. Un fardeau, d'ailleurs, alourdi par le peu d'estime qu'ils ont pour l'Angleterre:

Fuckin failures in a country ay failures. It's nae good blamin it oan the English fir colonising us. [...] We are colonised by wankers. We can't even pick a decent, vibrant, healthy culture to be colonised by. No. We're ruled by effete arseholes. What does that makes us? The lowest of the fuckin low, the scum of the earth. The most wretched, servile, miserable, pathetic trash that was ever shat intae creation. Ah don't hate the English [...]. Ah hate the Scots. (Welsh, 1996a [1993], p. 78) 
Steve Bell, renommé illustrateur écossais pour The Guardian, témoigne aussi de ce problème d'estime de soi dans l'Angleterre thatchérienne: "[1]es Écossais étaient des perdants, les jeunes étaient des perdants, les gens au chômage étaient des perdants" (Edemariam et Scott, 2009, n.p.; ma trad.).

Une telle conjoncture mène également à une transformation radicale de la sous-culture de la drogue, qui se vide complètement de l'essence subversive et culturelle propre aux années 1960-1970, où les hippies consommaient pour communier «au-delà de la frontière hautement symbolique érigée par la société "droite" (Willis, 1976, p. 107; ma trad.). En effet, dans le cas de l'Écosse et des toxicomanes de Welsh, la drogue est une fin en soi. Elle n'est ni symbolique ni contestataire, seulement physique. Les héroïnomanes écossais fuient leurs soucis (d'argent, personnels, et, circulairement, de drogues) en tripant. C'est un phénomène tellement courant que, dans les années 1980-1990, Édimbourg est nommée «capitale européenne du VIH/SIDA» en raison de ses nombreux héroïnomanes qui se partagent leurs seringues (Hunt, 1994, n.p.; ma trad.).

Dans ce contexte, le «Whair's it yis come fae then » de Begbie est certes une représentation du scots, mais aussi de ce tourment identitaire que vit l'Écosse. Il est l'incompréhension et le repli sur soi, mais aussi l'appel à l'autre, qu'il soit entendu ou non. Et c'est là ce qu'il faut traduire.

\section{Considérations traductologiques}

Trainspotting offre (et a offert) du fil à retordre aux traducteurs et traductrices de la planète parce qu'il met en lumière les insuffisances de leur langue, ou du moins, les restrictions auxquelles elle est soumise. Michael Gardiner, dans «British Territory: Irvine Welsh in English and Japanese", explique par exemple que le japonais, linguistiquement, ne réussit pas à faire la distinction entre la Grande-Bretagne et l'Angleterre, une subtilité pourtant essentielle dans un roman où les personnages vivent des sentiments compliqués par rapport aux identités écossaise, anglaise et britannique: "Ah've never felt British, because ah'm not. It's ugly and artificial. Ah've never really felt Scottish either, though. Scotland the brave, ma arse; Scotland the shitein cunt» (Welsh, 1996a [1993], p. 228). Le plus souvent, toutefois, ce sont les dictats du savoir-faire (ou du devoir-faire) éditorial qui nuisent aux traducteurs. Hilal Erkazanci, critiquant la version turque de Trainspotting, va jusqu'à parler de censure parce que le traducteur, Sabri Kaliç, «évite systématiquement 
de déranger le turc standard en collant à ses notions de rectitude syntaxique et lexicale»(2008, p. 248; ma trad.). La version espagnole de Federico Corriente, bien qu'elle joue avec les registres, n'arrive pas non plus à recréer la défamiliarisation linguistique de l'original (Ashley, 2010, p. 121). Eduardo Barros-Grela cerne deux possibilités qui pourraient expliquer les lacunes de cette traduction, soit «l'urgence éditoriale qu'on impose actuellement aux traducteurs en Espagne» (2003, n.p.; ma trad.) et le fait que «l'environnement culturel du traducteur ne lui donne pas les compétences nécessaires » (ibid.) pour bien comprendre les subtilités de la langue qu'il tente de dépeindre. Selon Meschonnic, traduire s'est installé dans une «situation babélienne» où l'on ne traduit que du sens, des mots, des phrases, des langues (1995b, p. 514). Or, traduire le sens, le fond, c'est négliger la voix du signe, celle de l'auteur et sa voix propre. Faute d'entendre la singularité du texte, faute d'entendre ce que les mots font, on crée des textes déjà vieux où, comme on se plaît à le dire, quelque chose «ne passe pas». Ainsi, lorsque Meschonnic affirme dans Éthique et politique du traduire que la Bible n'a pas encore été traduite en français, c'est parce que sa voix ne s'y est jamais retrouvée. Pourtant, la voix du texte littéraire est un élément essentiel et un complément indissociable de ce que dit le discours. Il est la façon de dire le discours. Il est le corps du discours qui, sans lui, n'en est que le squelette. Un cadavre, dirait Meschonnic. Pour lui, "plus que ce qu'un texte dit, c'est ce qu'il fait qui est à traduire; plus que le sens, c'est la force, l'affect" (Meschonnic, 2007, p. 55). Cette affirmation devient toutefois problématique lorsqu'on commence à écrire. Bien entendu, il est beaucoup plus facile de réfléchir à la voix, à l'oralité, lorsqu'on se trouve en présence de textes qui appartiennent à la tradition orale - Meschonnic luimême a travaillé à traduire la Bible, qu'il cherchait à «embibler»:

Embibler, c'est donc aussi, paradoxalement, déthéologiser, c'est-à-dire désémiotiser, défrançaiscourantiser ce langage. Qui n'a jamais été du langage courant. Pour retrouver la force du continu effacée par le travail des effaçantes, c'està-dire des traductions qui courent après le français courant pour faire du préchi-précha. (ibid., p. 148)

Meschonnic se positionne contre le français courant, contre le «traduire-désécrire» (ibid., p. 71), stratégie largement acceptée en traduction qui consiste à puiser dans les formules convenues, dites idiomatiques, de la langue. Pour ma part, le Trainspotting d'Irvine Welsh alimente ma réflexion parce qu'il part d'une langue qui se 
veut la représentation écrite de la langue parlée de l'Édimbourg héroïnomane des années 1980-1990, une parole qui veut signifier sa présence au monde, qui veut se faire entendre. Cette langue, c'est la voix, au sens meschonnicien, de Trainspotting. C'est par cette voix que passent l'abandon, l'incompréhension, le repli sur soi de l'œuvre. C'est par cette voix multipliée par huit que l'on comprend toute la détresse, l'humour et l'horreur de l'Écosse d'Irvine Welsh. C'est également cette voix qui crée le choc et qui permet l'ouverture d'un dialogue entre l'œuvre et le lecteur.

En réfléchissant à la voix, dit Meschnonic, on est forcé d'inventer une façon de penser le langage qui n'est pas fournie par notre tradition culturelle, qui «essentiellement est une tradition du discontinu qui consiste à penser le langage en termes de langue, de mots, de sens avec des formes et des phrases, toutes unités qui sont des formes discontinues» (1995a, 2:32), une vision confirmée, peut-être malgré lui, par Umberto Eco, qui affirme traduire phrase par phrase (2006, p. 245). Meschonnic n'y va pas de main morte lorsqu'il critique les dictats de la traduction littéraire actuelle, qu'il considère folie pure, voire schizophrénie, parce qu'ils s'évertuent à réduire la traduction au simple exercice d'un passage d'informations entre deux pôles, exercice lui-même enfermé dans le dualisme contraignant la traduction à choisir soit le sens, soit la lettre.

Ce qui est mis en cause, comme le dit Stéphane Bédard, «c'est la propension à faire passer un discours à fonctionnement oral à un texte marqué par les conventions de l'écrit. [...] Dès lors le discours ne dit plus: il "veut dire” (1995, p. 571). Le traducteur qui accorde trop d'importance au signifié risque de perdre, dans cet élan, le signifiant, stratégie qui est d'usage en pragmatique, mais qui se révèle destructrice en traduction littéraire. Pour Meschonnic, la plus petite unité de sens du texte, c'est le texte lui-même. Historiquement, le traducteur a dissimulé son manque d'écoute du texte par un embellissement des mots, comme en témoignent les Belles Infidèles. Le texte ainsi traduit devient énoncé et non énonciation: il ne fait plus.

Meschonnic ne le dira jamais assez: le traducteur n'est pas qu'un passeur. Le traduire est un exercice de réécriture qui nécessite une sensibilité d'écrivain et une écoute. En cela, le traducteur n'est pas qu'un Charon dans sa barque; il ne doit pas que faire passer le voyageur, il doit aussi être le voyageur, parler avec le voyageur et le questionner. Ainsi, 
dès qu'on lit sans question, on ne lit plus, on est, à l'inverse, dévoré par l'«objet» de la lecture. C'est bien alors qu'au lieu d'un sujet de la lecture, il n'y a plus que la créature d'un réseau grammatical, le réseau d'une logique plus puissante et qui a installé son monde. (Meschonnic, 1989, p. 114)

Tomber dans l'envie de plaire à son lecteur, dans le désir de faire beau, c'est masquer mieux la surdité que le traducteur doit transcender. Il ne fabrique pas du sens, il ne peut pas faire dire ce que le texte fait, parce que

démontrer uniquement la production et les modes d'organisation du sens, d'où le sujet est d'emblée évacué et déresponsabilisé, est à l'opposé du rapport d'écoute avec le texte, écoute qui englobe l'intonation, le corps, la voix et la vie, tout «ce qui échappe au sens, c'est-à-dire au modèle du signe». (ibid., p. 203)

$\mathrm{Ne}$ traduire que du sens, donc, c'est ne pas avoir de voix. Selon Meschonnic, il faudrait plutôt écouter le rythme, pas seulement le rythme de la langue, mais aussi le rythme poétique, qui fait que chaque texte est rythmé à sa manière, qui fait qu'une langue est bien plus qu'une langue de grammaire et de lexique.

\section{Quand traduire rime avec taire: les traductions françaises de Trainspotting}

Or, la voix "agressivement écossaise, et parfois intransigeante» (Ashley, 2010, p. 113; ma trad.) de Welsh est précisément ce qui ne passe pas dans les traductions existantes en français. La langue de Welsh, dans toutes ses imperfections, est systématiquement lissée, ce qui entraîne un renversement du discours de l'œuvre, effaçant le commentaire sur la langue au profit d'une critique sur les classes sociales (qui soit dit en passant est déjà présente dans l'œuvre), inversant du tout au tout les rapports de force entre les personnages et simplifiant à l'excès une langue qui a été façonnée dans le but de défamiliariser le lecteur, d'être difficile à déchiffrer. Revenons à la scène du train avec Begbie abordée plus haut. Dans le train vers Londres, il est assis à côté de deux Canadiennes:

- No fuckin shy, they British Rail cunts, eh? ah sais, nudgin the burd next tae us.

- Pardon? it sais tae us, sortay soundin likes, 'par-dawn' ken?

-Whair's it yis come fae then? 
- Sorry, I can't really understand you... These foreign cunts've goat trouble wi the Queen's fuckin English, ken. Ye huv tae speak louder, slower, $n$ likesay mair posh, fir the cunts tae understand ye.

— WHERE... DO ... YOU... COME... FROM?

That dis the fuckin trick. These nosey cunts in front ay us look roond. Ah stares back at the cunts. Some fucker's oan a burst mooth before the end ay this fuckin journey, ah kin see that now.

— Ehm ... we're from Toronto, Canada.

- Tirawnto. That wis the Lone Ranger's mate, wis it no? ah sais. The burds jist look it us. Some punters dinnae fuckin understand the Scottish sense ay humour. (Welsh, 1996a [1993], p. 114-115)

La présence de la voix dans cet échange permet de créer des rapports de force entre les personnages qui cimentent le sentiment d'infériorité et d'incompréhension des Écossais par rapport à l'épicentre culturel et économique qu'est Londres et, par extension, par rapport à l'Anglophonie en général. Ainsi, dans la scène, on sent bien que Begbie est agacé par l'incompréhension des deux Canadiennes, lui qui parle pourtant le «Queen's fuckin English». Le rapport de force est clair: il parle un anglais inférieur, écrit phonétiquement, qui mine la compréhension des autres. De leur côté, les Canadiennes, malgré quelques différences linguistiques dans la prononciation de «Toronto " (Tirawnto») et de «Pardon» («Par-dawn»), sont parfaitement bien comprises, et leur dialogue est rédigé en anglais standard. L'effet créé est tragicomique: on devine bien que, malgré l'hilarité de la scène, le fait de ne pas être compris est un des drames de la vie de Begbie, ainsi que sa prison.

Eric Lindor Fall, dans sa traduction publiée en 1996 aux éditions de l'Olivier, fait tout autrement et prend le pari de rendre Begbie dans un français familier, mais grammatical. En flagrant contresens, ce sont maintenant les Canadiennes qui parlent en phonétique! Dans le chapitre curieusement intitulé "Caca dedans", Begbie s'exclame:

- Putain, ils manquent pas d'air, ces cons du British Rail, hein? je fais en coudoyant dans le flanc de la caille posée à côté de moi.

- Je vous demande pardon? qu'elle me fait et ça sonne genre « démainde pairdon ». 
- Attends, toi, tu viens d'où?

- Je rgraitte, je ne vous comprin pas bien...

Les cons qui viennent d'ailleurs ont de vrais problèmes avec notre putain d'anglais anglais. Pour que ces cons prennent, il faut leur parler fort, lentement et surtout façon je ne suce que des virgules épluchées avec des gants.

— PUIS-JE... SAVOIR... D'OÙ...TU... VIENS...?

C'est le seul moyen, putain. Et les connards de devant qui se retournent pour mater. Je leur douche le cul d'un seul regard. Y a un enculé qui va se faire éclater la bouche avant la fin de ce foutu voyage, je le vois d'ici.

— Ehm... Nous sommes de Trointo, Canédé.

— Tonto. C'était le pote du Lone Ranger, non? je fais.

La caille me regarde. Y a des gens qui nont vraiment pas le sens de l'humour écossais. (Welsh, 1996b, p.135-136)

Ici, l'effet comique est modifié, parce que le rapport de force est neutralisé, et donc tout le propos change. Les Canadiennes deviennent les sujets de plaisanterie, celles qui s'expriment de façon incompréhensible, alors que Begbie, dans une langue par comparaison tout à fait correcte, se moque d'elles. Par l'intermédiaire de ce qui semble une parodie du français québécois, Lindor Fall inverse complètement le propos de l'œuvre. L'inconscience linguistique de Begbie, qui en anglais lui donne l'audace de se moquer de l'accent des Canadiennes, est également perdue.

$\mathrm{Si}$ Katherine Ashley, auteure du chapitre «Welsh and Translation» du Edinburgh Companion to Irvine Welsh de Berthold Schoene, fait les mêmes observations que moi, à savoir que les rapports de force du roman sont renversés dans la traduction française de Lindor Fall, elle refuse néanmoins de les qualifier de préjudiciables. Au contraire: pour elle, le texte original et les traductions doivent remplir deux fonctions différentes. L'original est «hétéroglossique», "fracture» l'anglais et crée «un débat sur les discours majoritaires et minoritaires ", alors que le Trainspotting traduit, même s'il fait l'objet d'une standardisation de la langue dans la traduction, "ajoute une couche de sens» qui permettrait aux «lecteurs étrangers de voir l'œuvre d'un point de vue mondial plutôt qu'écossais" et au roman, "emprisonné dans sa propre situation linguistique ", d'être libéré et de "participer à l'hégémonie culturelle et de la confronter tout à la fois » (Ashley, 2010, p. 125; 
ma trad.). Finalement, bien qu'elle admette que cela puisse être, au choix, aliénant ou libérateur, elle prend soin de mentionner que la traduction a l'avantage de permettre à Irvine Welsh d'être vu (et lu) «comme un auteur, plutôt que simplement comme un auteur écossais» (ibid.; ma trad.; c'est Ashley qui souligne), d'être reconnu de part et d'autre de la planète littéraire. Il semble pourtant clair que c'est l'intrinsèque scottitude de Welsh, justement, qui lui a permis d'obtenir le statut de superstar de la littérature, et que cette identification à l'Écosse ne peut (et ne devrait pas) être dissociable de son œuvre. Par conséquent, le fait de saluer la mondialisation de Trainspotting est parfaitement absurde.

Quinze ans plus tard, en 2011, Le Seuil et le traducteur JeanRené Étienne ressentent le besoin de retraduire Welsh, le texte de Lindor Fall étant truffé d'erreurs, de faux sens et de contresens. Dans la traduction de 2011, Jean-René Étienne évite de renverser le propos, mais il ne marque pas non plus la différence de langue entre les Canadiennes et Begbie. Dans le chapitre «De capitale à cacapitale», on lit ceci:

- Y se goinfrent bien, ces enculés de British Rail, hein? j'y dis, en donnant un ptit coup de coude à la nana d'à côté.

— Pardon? qu'elle me fait, mais ça sonne «par-dawn» ou quoi.

- Mais ouais, mais d'où tu viens?

- Désolée, je ne comprends pas très bien ce que vous dites. C'est quoi ces connasses de métèques quont du mal avec l'anglais de la Reine. Faut leur parler plus fort, plus lentement, et comme qui dirait plus bourge pour qu'elles te comprennent.

— D'OÙ...VIENS...TU ?

C'est ça qu'il lui fallait. Et les fouille-merde de devant qui se retournent pour mater, jles toise. $Y$ a un enculé qui va se faire péter la gueule avant la fin du voyage, jle sens.

— Euh... On est du Canada, de Toronto.

- Tirawnto. C'était le pote à Lone Ranger ça, non? j’y dis. La nana me regarde, y en a qui pigent rien du tout à l'humour écossais. (Welsh, 2011, p. 152-153)

Dans le cas présenté ci-dessus, non seulement l'humour d'Irvine Welsh tombe à plat, mais la colère de Begbie, tout comme l'incompréhension des Canadiennes, sont inexplicables. Le rapport de force est complètement anéanti. 
Trainspotting donne à lire un commentaire sur les classes sociales, mais également à un discours sur la langue. Tout au long du roman, c'est l'accent des personnages qui les identifie comme Écossais. C'est l'accent ou l'absence d'accent qui leur permet de passer incognito ou non, de subir ou non la pression et la honte de leurs racines, d'être compris ou non, d'être estimés ou non. En effet, les nombreux personnages du roman, universitaires et ouvriers confondus, s'expriment tous dans une langue également non standard. Ce qu'il faut en comprendre, c'est que le sociolecte scots n'est pas qu'une affaire de classe, mais aussi de culture, et que peu importe le niveau d'éducation, l'accent scots a toujours des répercussions sur la vie des personnages. Dans le contexte politique du roman, ces répercussions sont souvent négatives. La compétence linguistique, celle qui permet de naviguer entre différents registres, entre différentes identités, décide de la liberté ou de l'absence de liberté des personnages. Elle fait toute la différence dans chacune des interactions des personnages du roman. Les traductions françaises, en ne marquant pas l'orthographe, en taisant le sens que cette orthographe porte, évacuent le rapport à la langue vécu par les personnages. Elles vont également à l'encontre de l'intention qu'avait Irvine Welsh en écrivant son roman. En effet, le sociolecte utilisé par Welsh n'a pas pour but de frustrer le lecteur, mais de marquer l'œuvre comme autre. Elle est lourde de sens. Sa densité est un moyen de résister aux "normes linguistiques et textuelles qui codifient (et qui ont colonisé) la littérature écossaise » (Ashley, 2010, p.119; ma trad.). Il s'agit également d'une «déclaration d'indépendance envers les pouvoirs littéraire, culturel et politique symbolisés par le Charlotte Square d'Édimbourg» (ibid.; ma trad.), qu'il associe à la bourgeoisie pro-langue anglaise et pro-Union. Cette marginalisation est représentée et renforcée par la langue sans compromis ni explication que Welsh livre à l'intelligence et à la patience des lecteurs.

On peut expliquer les défauts de la traduction de 1996 par le fait que son traducteur avait la lourde tâche de présenter Welsh, alors un parfait inconnu, et son monde au public français. Ces traductions, selon Meschonnic, sont souvent des traductionsintroduction, définies par le "possible d'une époque», soit la somme des idées reçues (ce qui peut être lu, écrit, et traduit ou non) d'une époque (1973, p. 320-321). La traduction-introduction, contrairement à la traduction-texte qui est œuvre à part entière et vivante dans la culture d'accueil, se caractérise par une certaine 
prudence et une surabondance de notes et est condamnée à (mal) vieillir parce qu'elle n'est pas texte; elle ménage un nouvel espace dans la littérature d'accueil. Une fois l'introduction faite, la voie est ouverte à la traduction-texte (une retraduction) qui, délestée de ce mandat de présentation, peut devenir œuvre. La retraduction de 2011, "meilleure» que la précédente, devrait donc être la traduction-texte: l'auteur en effet n'est plus à présenter, son œuvre fait figure de culte et on annonçait déjà, à l'époque, la possibilité d'une suite à l'adaptation cinématographique signée Danny Boyle. ${ }^{3}$ En n'élucidant pas l'énigme du marquage phonétique du sociolecte de Welsh, toutefois, ni Étienne ni Lindor Fall ne retrouvent la voix de l'original, et l'Écosse, ce faisant, reste incomprise parce que dénaturée. La traduction-texte de Trainspotting, ainsi, n'existe pas encore.

\section{Le sociolecte québécois pour rompre avec la "pathologie de l'universalisme"}

L'utilisation d'un sociolecte à l'écrit est loin d'être anecdotique. $\mathrm{Au}$-delà du sociolecte des linguistes, qui n'est que la description de phénomènes linguistiques observables dans le monde réel, son utilisation en littérature revêt un sens souvent politique, qu'il recrée les rapports de force déjà existants au sein d'une société ou qu'il soit revendicateur. Dans le théâtre classique, notamment, il arrivait qu'on fasse parler les personnages de paysans dans une langue abâtardie qui les faisait paraître simples et crédules, voire benêts, et toujours à la merci des seigneurs au verbe gracieux et à l'esprit vif. Molière l'utilisait dans un but similairement parodique dans Dom Juan, où les paysans servaient l'effet comique en tant que boucs émissaires et confortaient la bourgeoisie (le public de Molière) dans sa supériorité (linguistique). Lorsque Dom Juan devient violent, toutefois, on assiste à l'appauvrissement momentané de sa langue (à coup d'onomatopées et d'expressions utilisées préalablement par les paysans). Le sociolecte, ainsi, sert également à montrer la régression vers la violence du personnage, à le rapprocher des paysans et à le montrer dans son plus simple appareil: peu réfléchi, sans élégance et violent.

À l'opposé, le sociolecte écrit a aussi joué un rôle dans la lutte pour la légitimité linguistique de bien des cultures et langues minoritaires dans le monde, notamment celles du Québec.

3. On sait que Trainspotting 2 a pris l'affiche en 2017, presque exactement vingt ans après l'original. 
Comme l'a signalé Annie Brisset dans Sociocritique de la traduction, l'utilisation d'un sociolecte québécois, le joual, a permis aux auteurs de se défaire de leur insécurité linguistique, de parler à leur peuple et de faire de leur langue, longtemps brimée et ridiculisée (Bouchard, 1988 , p. 9), non seulement un instrument au service de l'art, mais le véhicule d'un mouvement politique. En traduction théâtrale, explique Brisset, traduire "n'a plus pour objet de transmettre le discours de l'Étranger mais d'utiliser l'Étranger pour cautionner son propre discours, celui de l'émancipation nationale» (1990, p. 312). De la même manière, Martin Bowman, qui a traduit les Bellessœurs de Michel Tremblay en utilisant la langue vernaculaire des Lowlands écossaises, affirme que «même la mention d'une langue vernaculaire est un acte politique. Tout comme, bien entendu, la traduction dans une telle langue» (2000, p. 27; ma trad.). Chez Molière et Bowman, tout comme chez les auteurs québécois décrits par Brisset, le sociolecte revêt une grande importance politique et transmet un message qui dépasse ce qui est écrit ${ }^{4}$.

Trainspotting s'inscrit sans contredit dans un mouvement identitaire semblable. La voix de l'Écosse, enfin, est diffusée à grande échelle. Cette diffusion se révèle particulièrement importante pour les écrivains écossais de l'époque qui, comme l'explique Robert Crawford, s'affairent «à créer une voix littéraire distincte de celle de l'Angleterre, un but que certains atteign[ent] par l'incorporation du scots comme véhicule non seulement des dialogues, mais de la narration elle-même» (2000 [1992], p. 221; ma trad.). Cette stratégie d'écriture, décrite par Crawford comme une décentralisation de la littérature anglaise, ne s'effectue d'ailleurs pas qu'au Royaume-Uni, mais dans toutes les anciennes colonies anglaises, qui «marquent leur appartenance à un espace non central, non métropolitain et postcolonial» (ibid.; ma trad.), espace qui les différencie de la littérature anglaise dite standard. L'impact de l'écriture «non anglaise» en anglais est d'autant plus important qu'il a connu un succès mondial avec Trainspotting. Le scots dans Trainspotting s'oppose ainsi à l'idée traditionnelle et historique d'une langue orale sans autorité et «inapte pour la littérature en

4. Le théâtre, parce qu'il est soumis à des critères de jouabilité et de performativité qui forcent le texte à être immédiatement lisible pour l'acteur et compréhensible pour le spectateur, n'offre pas la solution à l'énigme du sociolecte dans Trainspotting. Il n'est donc pas abordé en profondeur dans cet article, même si les dramaturges sont les champions de la traduction des sociolectes. 
raison de sa "bassesse", de sa "vulgarité" et de son "impropriété" » (ibid., p. 223; ma trad.). Ce qu'Irvine Welsh fait, c'est étendre les possibilités de la langue au-delà du simple exotisme - qui se résume souvent à l'utilisation de quelques adjectifs «colorés» et noms «inhabituels» - pour en faire un moyen de communication à part entière et qui participe au récit. Ce faisant, Crawford voit en Welsh la permission d'utiliser le scots non seulement pour exprimer «le lyrisme d'une conscience déjà aliénée, mais aussi [...] les préoccupations quotidiennes dans le roman» (ibid., p. 222; ma trad.).

Trainspotting offre à lire une langue coup de poing, qui se distingue et qui permet la naissance d'une nouvelle littérature dans la langue anglaise. Ce nouvel espace identitaire ne fait certes pas l'unanimité, mais cela ne semble pas effrayer Irvine Welsh: "C'est ce que mes livres font, [ils] divisent les gens et les jurys, et c'est une bonne chose. En tant qu'auteur, si j'arrive à faire grincer quelques dents, alors j'ai laissé ma marque, j'ai accompli quelque chose d'utile» (cité dans Peddie, 2007, p. 132; ma trad.). L'arrivée de Welsh comme porteur d'une nouvelle réalité a certainement créé des remous. Le travail du traducteur, donc, consisterait à reproduire ce remous.

Alors, qu'est-ce qui pourrait bien expliquer ce systématique effacement par les traducteurs parisiens? D'aucuns seraient tentés d'évoquer l'incompatibilité des langues. Le problème de la différence des langues, c'est vrai, est reconnu des traducteurs depuis longtemps. Les langues, il s'agit d'un fait reconnu, ne s'équivalent pas. Ce concours de circonstances ne signifie évidemment pas qu'il faille s'abandonner à la notion inexorable d'intraduisibilité, mais plutôt qu'il faille peut-être chercher à la contourner, à trouver ailleurs la solution à cet épineux problème. Une chose est sûre, la langue de Trainspotting n'existera pas tant et aussi longtemps qu'on l'académisera. Le français normalisé en vigueur dans le monde de l'édition hexagonale n'est pas, à mon avis, apte à rendre la réalité du scots, ce qui ne veut pas dire que le français d'ailleurs, comme le français québécois, ne saurait pas le faire. Michel Tremblay trouvait entre les États-Unis et le Québec des affinités facilitant son travail de traduction-adaptation. Une question de "parenté naturelle,» dit Sathya Rao (2013, p. 74), sans doute tributaire de la proximité géographique des deux cultures (Louise Ladouceur parle d'une "parenté nord-américaine» [2006, p. 24]). Cette parenté s'explique, 
selon Annie Brisset, par le fait que «le théâtre américain offre une matrice thématique dans laquelle peuvent se couler les grandes préoccupations qui traversent la société québécoise» (1990, p. 50). Par ses traductions de l'américain en québécois, Michel Tremblay aurait ainsi «ménagé dans le système théâtral du Québec une ouverture » qui n'existe pas en dramaturgie française institutionnelle et qui «a étendu la traduisibilité des sociolectes de la dramaturgie anglo-américaine, sociolectes qui trouvent un équivalent naturel dans la culture québécoise, mais non pas dans la culture française» (ibid.). Comme le note Sathya Rao, certains chercheurs, Louise Ladouceur en tête, vont même jusqu'à faire du joual (ou du français québécois) "le "véhicule" par excellence de cette identité étasunienne reterritorialisée au Québec» (2013, p. 74), un véhicule qui ne se réduit toutefois plus aux « excès ethnocentristes des années 1970 » (Lavoie, citée dans Rao, 2013, p. 73). Cette compréhension naturelle, dit Rao, pourrait aussi bien être réelle que fantasmée dans le cas des États-Unis, malgré un fond culturel commun, parce qu'elle est unidirectionnelle. Le cas de l'Écosse se distingue particulièrement parce qu'il implique un mouvement bidirectionnel avec le Québec: l'Écosse est couramment traduite au Québec et vice-versa. La parenté naturelle, ainsi, traverse l'Atlantique. ${ }^{5}$

L'Écosse, nation jumelle du Québec? Difficile à dire, certes, mais les similitudes sont légions. Évidemment, le Québec n'est pas la seule nation (ou le seul groupe) francophone qui pourrait se trouver des affinités avec l'Écosse ou qui serait à même de la traduire. L'idée, c'est de se distancier des exigences de la traduction de masse centralisée en un seul endroit: il n'y a pas que Paris pour traduire l'Europe en français. Penser le contraire promeut l'érosion culturelle et linguistique. Lorsque toutes les traductions de langue française sont centralisées en France, la pluralité culturelle ne peut s'épanouir. Dans «The Cracked Looking Glass of Servants: Translation and Minority Languages in a Global Age», Michael

5. Tremblay détient d'ailleurs un doctorat honorifique de l'Université de Stirling, en Écosse, pour sa contribution exceptionnelle au théâtre écossais (Delisle et Woodsworth, 2012), bien qu'il n'ait jamais signé une œuvre dans la langue de Shakespeare. Bowman et Findlay, traducteurs des Belles-seeurs et de sept autres pièces de Tremblay, ont également traduit en écossais d'autres auteurs québécois comme Dominic Champagne, Michel-Marc Bouchard et Jeanne-Mance Delisle (ibid.). Avec le dramaturge Wajdi Mouawad, Bowman a également traduit l'adaptation théâtrale de Trainspotting en français québécois. 
Cronin affirmait que cette tendance à la normalisation en vigueur dans les milieux pragmatiques créait, dans le contexte actuel de mondialisation, une "minorisation" de toutes les langues» (1998, p. 145; ma trad., c'est Cronin qui souligne) au profit de la langue majoritaire (dans son exemple, l'anglais; dans le mien, le français). Le besoin d'absolument se comprendre, dit-il, crée une "pathologie de l'universalisme» (ibid., p. 155; ma trad.) qui force ou pousse les locuteurs à se rassembler autour d'une seule langue, ou d'une seule variété de langue, pour atteindre l'idyllique communication interculturelle. Il y a lieu de se demander à quel prix s'accomplit cette utopie. Dans Translation and Globalization, Cronin semble offrir une réponse à cette interrogation en amenant le concept de "clonialism», jeu de mots alliant les termes colonialisme et clone, qu'il explique ainsi:

Différents pays, différents continents, mais les mêmes McDonald's, les mêmes épisodes de Dallas et de Friends, les mêmes films de Disney dans les mêmes multiplexes, les mêmes Microsoft Windows et les mêmes Britney Spears. La mort de la diversité se joue dans la propagation du double. (2003, p. 128 ; ma trad.)

Le fait de créer ces clones, de ne jamais bousculer la langue, de forcer sa compréhension, de la babéliser, rend la littérature a-culturelle, dans le sens où elle finit par engendrer des produits mondiaux accessibles, mais fabriqués, qui prennent dans l'imaginaire collectif la place des littératures dominées. Uniformiser et standardiser la langue française, c'est ne permettre qu'une seule identité francophone, qui plus est dictée par des normes éditoriales de fluidité, d'élégance et de rapidité. Les identités autres, étranges et étrangères, doivent trouver leur place dans le paysage linguistique, littéraire et éditorial. C'est le devoir des langues majoritaires de leur laisser une place.

Dans un contexte où l'on voudrait traduire un texte comme Trainspotting, il est évident qu'il faut agir sur la langue et rompre avec la norme du français écrit hexagonal (à laquelle les auteurs québécois se pliaient jadis dans l'espoir d'être mis dans la course au Goncourt [Brisset, 1990, p. 245]) qui ne se concilie pas à un texte graphiquement hors-norme comme Trainspotting. Pour le traduire, en essence, il faut décentraliser le français (ou le "déterritorialiser" [Deleuze et Guattari, 1975, p. 30]) vers un «autre» non-français, l'enlaidir, le déraciner, l'abâtardir, pour le rendre capable d'exprimer 
des réalités ni belles ni françaises ni standard ${ }^{6}$. L'idée n'est pas d'inventer une langue de toute pièce - «inventer une langue, avertit Françoise Morvan, serait fausser les données du jeu et risquer au surplus d'être incompréhensible» (1994, p. 65) -, mais de recréer une langue crédible, une représentation en laquelle le lecteur a confiance pour lui permettre d'en découvrir les règles et de traverser l'épreuve initiale de la lecture. La voix de Welsh, dans Trainspotting, est un système: Welsh avoue lui-même écrire son texte en anglais écossais plutôt standard, qu'il modifie graduellement (Edemariam et Scott, 2009, n.p.). Welsh n'a pas copié un sociolecte, il l'a réinventé, travaillé, retravaillé, de manière à le rendre audible aux lecteurs.

Le choix d'une variante du français québécois est fortement informé par ce fait; il s'agit d'un sociolecte que je connais, que je maîtrise et avec lequel je peux jouer. Le français québécois me permet, par son contact avec l'anglais, de rapprocher le français traduisant de l'anglais traduit et d'atteindre un même niveau de langue. Le contexte sociohistorique du Québec, fort de similitudes assez prenantes avec l'Écosse sur les plans politique, économique, linguistique et culturel, guide également mon choix. Le français québécois, enfin, s'oppose à ce qui a été fait dans les traductions hexagonales qui ont choisi de ne pas garder le codage orthographique et la coloration du sociolecte dans le texte d'arrivée, et s'inscrit dans la foulée de ce que Judith Lavoie propose pour la traduction $\mathrm{du}$ vernaculaire noir américain de The Adventures of Huckleberry Finn (1994) et de ce que le Groupe de recherche en traductologie (GRETI) de l'Université McGill a fait dans son propre exercice de traduction avec Faulkner. Comme le dit Meschonnic, le langage fait autant qu'il dit, et c'est le grand défi de la traduction du discours d'une œuvre. L'objectif de la poétique de Meschonnic est de trouver (sinon de réfléchir à) comment. Selon lui, les traducteurs nont plus besoin de disparaître derrière leurs traductions. C'est dans cette

6. Ce qui ne veut pas dire que le français de France (non normé, soit-il ch'ti ou marseillais ou autre) ne sache pas faire, ce que Françoise Morvan a prouvé en utilisant le berrichon dans une pièce d'Eugene O'Neill. Cela étant dit, n'étant ni marseillais, ni ch'ti, je me résous à utiliser ma langue, le français vernaculaire montréalais, car force est d'admettre qu'il n'y a pas plus dangereux que de s'aventurer à traduire dans une langue ou un sociolecte que l'on ne connaît pas, et que même les recherches les plus poussées ne permettraient probablement pas d'atteindre la sensibilité nécessaire à son utilisation. 
optique que j'ai traduit l'extrait de Trainspotting: en cherchant à en rendre l'effet, que Meschonnic appelle la voix. Il n'est donc pas question du concept vétuste du mot-à-mot, car il ne s'agit pas de rendre la terminologie, mais la portée, l'expressivité et la spontanéité du texte de Welsh.

Lutilisation d'un sociolecte comme outil de traduction n'est toutefois lui-même pas sans risque, comme l'indique Françoise Morvan (1994). En effet, le traducteur pourrait faire du sociolecte sa panacée, la réponse à tous les maux. Welsh, après tout, a créé une langue qui «n'est d'aucune façon réellement ou historiquement scots, [...] pas plus qu'elle ne fait qu'épeler des mots anglais de manière à reproduire la façon dont leur prononciation par les personnages sonne aux oreilles de l'auteur» (Morace, 2001, p. 27; ma trad.). L'exercice de traduction auquel je me suis adonné ne consistait donc pas à faire l'archéologie du français québécois écrit pour ensuite en déguiser les mots d'Irvine Welsh, mais plutôt à tenter de retrouver, par le français québécois, les possibilités créatives engendrées par cette "défrançaiscourantisation» de la langue et cette lutte contre la neutralité culturelle. Les personnages ne parlent pas seulement une langue "hors-norme», ils permettent "de forger les moyens d'une autre conscience et d'une autre sensibilité» (Deleuze et Guattari, cités dans Karnicky, 2007, p. 138). Leurs voix cryptées, la voix du texte qu'ils transportent, ne tiennent plus tant du sens. Elle est système. C'est ce système - qui semble indéchiffrable à première vue, mais qui se laisse apprivoiser au bout d'une trentaine de pages - qui est méthodiquement, rigoureusement, évacué dans les traductions françaises de 1996 et de 2011.

\section{En réponse à Berman: une nouvelle «systématique de la déformation"}

À partir des études sociolinguistiques et phonologiques de Dumas (1974 et 1987), Bento (1998), Ostiguy et Tousignant (2008 [1993]), Paradis et Dolbec (2008) ainsi que Côté (2010), entre autres ${ }^{7}$, j'ai ciblé douze différences phonologiques et morphosyntaxiques $\mathrm{du}$ français montréalais (et, dans une

7. Plus de 36 études et ouvrages de sociolinguistique ont servi à la rédaction du mémoire de maîtrise duquel est inspiré cet article. Je ne nomme donc ici que les ouvrages en lien direct avec les différences abordées dans ces pages. À noter également que Paradis et Dolbec (2008) répertorient et synthétisent les résultats de plus de 129 autres études sociolinguistiques. 
exception, de la Beauce) par rapport au français hexagonal, que j'ai représentées graphiquement dans ma traduction: 1) le métaplasme, 2) la liaison fautive, 3) les particules interrogatives tu et -ti, 4) la particule là et 5) les anglicismes, qui constituent les transformations morphosyntaxiques; 6) l'affrication, 7) l'assimilation, 8) la simplification des groupes consonantiques et 9) l'affaiblissement, qui correspondent aux transformations apportées aux consonnes; 10) la diphtongaison, 11) les variations de prononciation selon l'aperture et le lieu d'articulation, 12) la fusion vocalique ainsi que 13) la nasalisation, qui correspondent aux transformations appliquées aux voyelles. Dans le présent article, faute d'espace, je n'aborderai que cinq de ces différences, à savoir l'affrication, la diphtongaison, le métaplasme, le marquage des variantes de prononciation et l'affaiblissement.

L'avantage de l'étude sociolinguistique est qu'elle informe sur les contextes d'apparition de ces comportements linguistiques, en plus d'identifier l'attitude des locuteurs par rapport à ceux-ci: ils sont tour à tour stigmatisés, à peine perçus, présents dans toutes les couches de la société, ou encore l'affaire des classes défavorisées ou peu scolarisées. Ces informations sont particulièrement utiles dans l'optique où Trainspotting met en scène huit narrateurs qui, bien que tous issus de la classe ouvrière, ont des compétences linguistiques et une éducation différentes. D'autres tendances observées en sociolinguistique peuvent également s'appliquer de façon plus générale aux personnages. Par exemple, Ostiguy et Tousignant affirment que les hommes manifestent plus souvent une indifférence à l'égard des variantes de registre: «[i]l appert qu'une certaine volonté de paraître "viril” ne serait pas tout à fait étrangère au comportement linguistique de certains représentants de la gent masculine» (2008 [1993], p. 19). Sans nécessairement chercher à genrer la langue des protagonistes, la désinvolture linguistique notée chez les hommes pourrait être imitée ou évitée selon la personnalité du personnage.

L'affrication est le phénomène qui consiste à prononcer une consonne à l'aide de deux consonnes, une occlusive et une fricative. En français québécois, ce phénomène touche la consonne /t/ devant les voyelles /i/ («i») et /y/ («u»), qui est ainsi prononcée [tsi] («tsi») et [tsy] («tsu»), ainsi que la consonne /d/, qui devient [dzi] («dzi») et [dzy] («dzu»). Dans la traduction que je propose, cette affrication est systématiquement marquée. Les adjectifs 
fortuné, ridicule, dur et le juron ostie deviennent, respectivement, fortsune, ridzicule, dzur et ostsie.

La diphtongaison, une des caractéristiques les plus frappantes du français québécois selon Dumas (1974, p. 25), désigne le changement de timbre d'une voyelle longue en cours d'émission. C'est ce qui fait qu'un Québécois, en parlant de sa génitrice, ne dira pas toujours mère $/ \mathrm{m} \varepsilon \mathrm{r} /$, mais parfois maïre ou méïre [mع $\varepsilon^{\mathrm{i}} \mathrm{r}$ ou $m \varepsilon^{\mathrm{j}} \mathrm{r}$ ]. Les voyelles intrinsèquement longues $/ \varepsilon /, / \mathrm{a} /, / \mathrm{o} /, / \mathrm{J} /$, notamment, sont systématiquement diphtonguées dans le texte traduit. La phrase "À mon âge, [il] faut savoir rester joyeux, même s'il pleut" devient: «À mon âoge, [y] faut savoère rester joéyeux, maïme s'y pleut». Malgré le fait que toutes les voyelles sont diphtongables en français québécois, il n'est pas toujours possible de les représenter à l'écrit sans utiliser les symboles de l'alphabet phonétique international. Dans l'optique où le codage doit obligatoirement se faire dans un alphabet connu du lecteur, certaines occasions de diphtongaison doivent être ignorées. C'est notamment le cas des

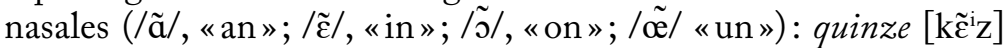
*quin-i-ze.

Le métaplasme désigne toute altération phonétique ou morphologique qui touche l'intégrité d'un mot. Il est souvent utilisé pour imiter les raccourcis de la langue parlée ou, anciennement, pour respecter les contraintes de la versification (encor'). On obtient le métaplasme de trois façons: par addition, suppression ou substitution. L'ajout d'un ou plusieurs phonèmes en début de mot s'appelle prosthèse. En milieu de mot, comme dans exiprès, pour exprès, c'est une épenthèse. Le paragoge, enfin, est un métaplasme par addition en finale de mot: bout, boutte. L'aphérèse a lieu en début de mot ('tention!), la syncope en milieu de mot (miman) et l'apocope, qui touche surtout les pronoms personnels ( $j e, m e$, te, le), en finale de mot. Le métaplasme par permutation, appelé métathèse, désigne l'interversion des phonèmes à l'intérieur d'une syllabe dans un mot ou à la frontière entre deux mots: vendredi devient vendeurdi, le gars devient eul'gars. Selon Paradis et Dolbec (2008, n.p.), la métathèse est associée au parler populaire.

Dans ma traduction, l'aperture des voyelles est marquée lorsqu'elle diffère de la prononciation standard. C'est un procédé qui est utilisé par Welsh aussi, et c'est ce que j’ai fait, entre autres, avec le fameux "a québécois" - qui se ferme dans la bouche des locuteurs - dans le syntagme pas pire beau gars: pâh pire beau gârs. 
La fermeture de /o/, l'ouverture de / $\varepsilon /$ («è») et la centralisation $\mathrm{de} / \mathrm{u} /$ («ou») sont également marquées: beaucoup devient boucoup, parfait est parfa' et nous-autres se transforme en nu z'autres.

Enfin, l'affaiblissement des fricatives est un phénomène articulatoire souvent associé aux locuteurs de la Beauce (mais que l'on trouve, selon Paradis et Dolbec (2008), dans toutes les régions du Québec), qui consiste à relâcher les fricatives post-alvéolaires, la sonore $/ \zeta /(« \mathrm{j} »)$ et la sourde $/ \mathrm{J} /($ (ch»), de façon à ne produire qu'un bruit de friction. En phonétique, cette friction est représentée par [h] ou [h], selon qu'elle est sonore ou non. Ainsi, bûcher devient bûher [byhe], et manger devient manher [mãhe]. Selon Paradis et Dolbec (ibid.), l'affaiblissement a surtout lieu dans le parler rural, chez les locuteurs masculins et dans les groupes sociaux moins favorisés. Dans ma traduction, je considère l'affaiblissement sonore comme la transformation orthographique la plus extrême. Elle est réservée à Begbie, le personnage à la langue la plus crue.

Afin de créer une unité dans la langue des personnages, j'agis sur l'orthographe par couche, ou degré, en appliquant d'abord les transformations morphosyntaxiques, l'affrication, le eye dialect et la simplification des groupes consonantiques finaux à tous les narrateurs. Cette stratégie me permet d'établir un «sociolecte de base». L'idiolecte de chacun se trouve dans les autres transformations, qui sont appliquées à divers degrés selon le personnage et qui sont consignées dans un tableau listant toutes les transformations possibles afin de mieux orienter mon codage. Ce processus d'intelligibilité est nécessaire et il reproduit une systématicité discursive qui assure la force des voix. Le fait de décider au préalable des transformations à appliquer au discours d'un personnage influence également mes tournures de phrases et me permet de créer, dans la traduction, des occasions de codage auxquelles je n'aurais pas nécessairement pensé autrement. Le tableau de Begbie (page suivante), par exemple, me rappelle d'utiliser la variante "éi» dans la diphtongaison de la voyelle $/ \varepsilon /$, la particule interrogative - $t i$ et l'affaiblissement voisé, entre autres, mais d'éviter l'assimilation voisée. 
Tableau 1. Tableau idiolectal - Begbie

\begin{tabular}{|c|c|c|}
\hline \multirow{7}{*}{ 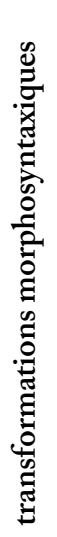 } & 1. Métasplasme & oui \\
\hline & 2. Liaisons fautives & oui \\
\hline & 3. Particules & \\
\hline & $\begin{array}{l}-t u \\
-t i\end{array}$ & $\begin{array}{l}\text { oui } \\
\text { oui }\end{array}$ \\
\hline & 4. Particule là & oui \\
\hline & $\begin{array}{l}\text { 5. Anglicismes } \\
\text { lexicaux } \\
\text { calques prépositionnels } \\
\text { double négation } \\
\text { élision que }\end{array}$ & $\begin{array}{l}\text { oui } \\
\text { oui } \\
\text { oui } \\
\text { oui }\end{array}$ \\
\hline & 6. Eye dialect & oui \\
\hline \multirow{4}{*}{ 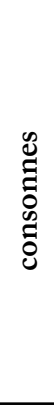 } & $\begin{array}{l}\text { 7. Affrications } \\
\text { /d/ } \\
\text { /t/ }\end{array}$ & $\begin{array}{l}\text { sonore } \\
\text { sourde }\end{array}$ \\
\hline & 8. Assimilation & $\begin{array}{l}\text { dévoisement: oui } \\
\text { voisement: non }\end{array}$ \\
\hline & 9. Simplification gr. conson. & $\begin{array}{l}\text { liquides, nasales et } \\
\text { occlusives }\end{array}$ \\
\hline & 10. Affaiblissement & oui, voisée \\
\hline \multirow{4}{*}{$\begin{array}{l}\frac{8}{0} \\
\stackrel{0}{0} \\
\frac{0}{5}\end{array}$} & 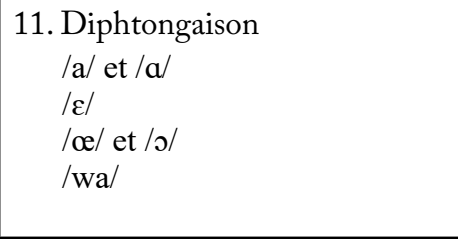 & $\begin{array}{l}\text { oui } \\
\text { variante «éi» } \\
\text { oui } \\
\text { variantes }[\mathrm{w} \supset][\mathrm{we}],[\mathrm{w} \varepsilon], \\
{[\mathrm{wa} \varepsilon]}\end{array}$ \\
\hline & $\begin{array}{l}\text { 12. Aperture et lieu d'articulation } \\
\text { fermeture } / \mathrm{a} / \mathrm{et} / \mathrm{a} / \\
\text { fermeture de } / \mathrm{o} / \\
\text { ouverture de } / \varepsilon / \\
\text { centralisation de } / \mathrm{o} / \\
\text { centralisation } \mathrm{de} / \mathrm{i} / \\
\text { fermeture de } / \mathrm{u} / \\
\text { centralisation de } / \mathrm{u} /\end{array}$ & $\begin{array}{l}\text { oui } \\
\text { oui } \\
\text { oui } \\
\text { oui } \\
\text { oui } \\
\text { oui } \\
\text { oui }\end{array}$ \\
\hline & 13. Fusion vocalique & partielle \\
\hline & 14. Nasalisation de $/ \varepsilon /$ & oui \\
\hline
\end{tabular}


Ainsi, dans le syntagme Je le sais (/zə lə se/), le «e» caduc dans le pronom personnel je tombe par apocope et est remplacé par une apostrophe: J'le sais [3ləsc]. Puis, j’inverse /lə/ par métathèse en / əl/: J'eul'sais [zolse]. Pour représenter l'affaiblissement du /z/, j'ai deux avenues, soit par l'aphérèse, en enlevant le « $j$ » complètement (Eul'sais), soit en lui substituant la voyelle glottale voisée /h/ («h»): H'eul'sais [həlse]. Dans le cas de Begbie (et selon son tableau), je sais que la deuxième option serait préférable. Finalement, afin de choquer l'œil davantage, je marque la prononciation du verbe sais par eye dialect: H'eul'sé [zolse].

Tableau 2. Degrés de transformations - Je le sais

\begin{tabular}{|l|l|l|}
\hline Phrase & Transformations & Degré \\
\hline Je le sais & Aucune transformation & 0 \\
\hline J_'le sais & Apocope du /ə/ & 1 \\
\hline J'eul'sais & Métathèse de /lə/ en /əl/ (permutation) & 2 \\
\hline _Eul'sais & Affaiblissement du /3/ (par aphérèse) & 3 \\
H'eul'sais & Affaiblissement du/3/(par substitution) & \\
\hline $\begin{array}{l}\text { Eul'sé } \\
\text { H'eul'sé }\end{array}$ & Eye dialect: simplification de l'orthographe & 4 \\
\hline
\end{tabular}

Toutes ces modifications créent un système à plusieurs niveaux qui s'adapte à tous les protagonistes du roman et qui mène à une traduction visant à recréer les rapports de force exprimés par Welsh ainsi que l'impact de l'original sur le lecteur:

- Sont câlissement pâh geinés, les crosseurs d'la British Rail, ein? heuj'dzis en donnant un coup d'coude à fille à côté.

— Pardon? qu'a m'dzit, pis c't'à crouére qu'a dzit «pardawn», t'sé?

- D'oussé qu'tsu viens coudonc?

- Désolée, je suis pas sûre de vous comprendre... Ah ben h'ai mon vouéyage, lé tsites tourisses ont d'la misère a'ec l'angla' d'la réine d'Angleterre, tabarnac. Faut leu' parler ben fort, ben lentement, pis ben fancé, tsu comprends-tsi, pour qu'è comprennent.

— D'OÙ... VIENS... TSU? 
C'tait çâh qu'y falla' pour qu'a l'allume. Y'âh dé z'osties d'fouines à marde a n'avant d'nous z'aut' qui s'eurtournent. Y vont s'manher deux, trois dents câssées avant 'a fin du vouéyage, h'eul'sens, estsie.

- Euh... On vient de Toronto, au Canada.

— Tirawno... c'tait pâh l'partner dzu Lone Ranger, çâh? heuj'dzis. Les deux morgarent, ben scotchées là. Eh boy, y'en a qui catchent pas pentoute heul'sens de l'humour écossa'.

Ici, le lecteur notera, en plus des anglicismes et de l'affrication, l'utilisation généralisée des métaplasmes (notamment l'éphenthèse du /1/ et la métathèse de /lə/), de la simplification des groupes consonantiques finaux (aut', leu') et de l'affaiblissement du /3/ par substitution (heuj'dzis, heul'sens). La fusion vocalique partielle est marquée dans a'ec et la diphtongaison, entre autres, dans crouère et vouéyage. Dans l'extrait, la voyelle/ع/subit trois transformations : la diphtongaison dans réine, la nasalisation dans geinés, et l'ouverture dans falla', angla' et écossa'. Le eye dialect, quant à lui, me permet de simplifier le déterminant les (lé), l'article des (dé) et le verbe savoir $\left(t^{\prime} e^{2}\right)$.

Pour traduire la portée politique de Trainspotting en français, il est important de reproduire l'effet que le roman a sur le lecteur anglais, dans toute son étrangeté et sa difficulté de lecture. En mobilisant les possibilités ré-énonciatrices du français québécois, je cherche à donner un souffle de vie à un texte qui, par deux fois, a été mis à mal par la moulinette du français normé. Ce faisant, je romps avec le discours de la fidélité qui fait de la traduction «un non-travail par rapport au texte de départ, une simple réplique», mais aussi avec le «topos de l'effacement du traducteur», deux notions néfastes parce qu'elles «occultent le réel» (Folkart, 1991, p. 11-12). La traduction, forcément, inévitablement, sera toujours autre. Cette altérité doit être assumée: Trainspotting en français québécois ne sera jamais écossais, pas plus que les traductions françaises ne l'étaient. Ce que j'essaie de faire, c'est de ne pas limiter le roman à une suite de portraits et tableaux trash comme les traductions parisiennes l'ont fait, et de recréer cet «autre» écossais qu'Irvine Welsh a su mettre au monde en anglais. Cet autre, j'en suis convaincu, ne peut exister en français que par un rigoureux codage de la langue, par sa décentralisation, qui reproduit les rapports de force entre le scots et l'anglais et, par extension, la force d'expression du scots, et qui permet de raconter l'histoire de 
ces personnages incompris et difficiles à comprendre. L'utilisation d'études sociolinguistiques témoignant des différences du français québécois par rapport au français hexagonal me permet d'ancrer ma pratique dans le réel et d'éviter l'arbitraire et la maladresse, qui auraient tôt fait de frustrer et de décourager le lecteur. Ici, traduire ne veut plus dire taire. Lexercice de la traduction que je mets en branle, plutôt, cherche à donner à l'auteur, dans une langue qui possède un système d'outils riches, rigoureux et personnalisables, la possibilité d'exister avec la même rage et la même force. Évidemment, la lutte contre la normalisation à outrance de la langue en traduction n'est pas gagnée d'avance, ni en France ni au Québec, et il y a fort à parier qu'il faudra attendre longtemps pour que les choses changent. En élaborant cette systématique de la déformation, toutefois, j'espère démontrer qu'il est possible, en français, d'utiliser le sociolecte comme outil de traduction rigoureux pour rendre la voix, au sens meschonnicien, d'une œuvre.

\section{Références}

Adorno, Theodor et Max Horkheimer (2002 [1944]). Dialectics of Enlightment. Palo Alto, Stanford University Press.

Ashley, Katherine (2010). «Welsh and Translation». In B. Schoene, dir. The Edinburgh Companion to Irvine Welsh. Édimbourg, Edinburg University Press, p. 113-125.

Barros-Grela, Eduardo (2003). «El tratamiento de lexicografía ficticia en la traducción de narrativa. Una perspectiva prosódico-discursiva en torno ala idiosincrasia sociocultural del traductor» [Le traitement de la lexicographie fictive dans la traduction du récit. Une perspective prosodico-discursive en ce qui a trait à l'idiosyncrasie socioculturelle du traducteur]. Espéculo. Revista de estudios literarios, 23. [http:// www.ucm.es/info/especulo/numero23/traducci.html] (consulté le $1^{\text {er }}$ décembre 2015).

Bédard, Stéphane (1995). "Cette sagesse qui bouscule». Meta, 40, 4, p. 564-578.

Bento, Margaret (1998). «Une étude sociophonétique des affriquées désonorisées en franco-québécois». Revue québécoise de linguistique, 26,1, p. $13-26$.

Berman, Antoine (1985). «La traduction et la lettre - ou l'auberge du lointain». In A. Berman et al., dir. Les Tours de Babel. Mauvezin, Éditions Trans-Europ-Repress.

Bouchard, Chantal (1988). "De la "langue du grand siècle" à la "langue humiliée": les Canadiens français et la langue populaire, 1879-1970». Recherches sociographiques, 29, 1, p. 7-21. 
Bowman, Martin (2000). «Scottish Horses and Montreal Trains. The Translation of Vernacular to Vernacular». In C.-A. Upton, dir. Moving Target. Manchester, St. Jerome Publishing, p. 25-33.

Brisset, Annie (1990). Sociocritique de la traduction: théâtre et altérité au Québec (1968-1988). Longueuil, Le Préambule.

Burzinsky, Andréa (2012). «Irvine Welsh talks Renton, Sick Boy and "Trainspotting USA"». Reuters, 17 septembre.

Chapdelaine,Annick(1996).«Reconstructions identitaires en traduction: le conflit des groupes et des langages dans The Hamlet de Faukner». Palimpsestes, 10, p. 93-114.

Chapdelaine, Annick et Gillian Lane-Mercier (1994). «Présentation. Traduire les sociolectes: définitions, problématiques, enjeux». TTR, 7,2, p. 7-10.

Côté, Marie-Hélène (2010). «La longueur vocalique devant consonne allongeante en contexte final et dérivé en français laurentien». In C. LeBlanc, F. Martineau et Y. Frenette, dir. Vues sur les français d'ici. Québec, Presses de l'Université Laval, p. 49-75.

Crawford, Robert (2000 [1992]). Devolving English Literature. Édimbourg, Edinburgh University Press.

Crawford, Robert (2009). Scotland's Books: A History of Scottish Literature. New York, Oxford University Press.

Cronin, Michael (1998). "The Cracked Looking Glass of Servants: Translation and Minority Languages in a Global Age». The Translator, 4, 2, p. 145-162.

Cronin, Michael (2003). Translation and Globalization. New York, Routledge.

Deleuze, Gilles et Felix Guattari (1975). Kafka. Pour une littérature mineure. Paris, Éditions de Minuit.

Delisle, Jean et Judith Woodsworth (dir.) (2012). Translators through History. Revised edition. Amsterdam/Philadelphie, John Benjamins.

Dumas, Denis (1974). «Durée vocalique et diphtongaison en français québécois ». Cahier de linguistique, 4, p. 13-55.

Dumas, Denis (1987). Nos façons de parler. Québec, Presses de l'Université du Québec.

Eco, Umberto (2006). Dire presque la même chose. Expériences de traduction. Paris, Grasset et Fasquelle.

Edemariam, Aida et Kirsty Scott (2009). "What Happened to the Trainspotting Generation?» The Guardian, 15 août.

Erkazanci, Hilal (2008). "Language Planning in Turkey: A Source of Censorship on Translation». In T. Seruya et M. L. Monis, dir. Translation and Censorship in Different Times and Landscapes. Newcastle, Cambridge Scholars Publishing. 
Folkart, Barbara (1991). Le conflit des énonciations: traduction et discours rapporté. Candiac [Québec], Éditions Balzac.

Gardiner, Michael (2003). «'British Territory': Irvine Welsh in English and Japanese». Textual Practice, 17, 1, p. 101-117.

Hunt, Liz (1994). «Aids Taking Heavy Toll of City's Drug Users: Edinburgh Feels Impact of HIV». The Independent, 4 août.

Karnicky, Jeffrey (2007). Contemporary Fictions and the Ethics of Modern Culture. New York, Palgrave MacMillan.

Ladouceur, Louise (2006). «Les voix de la marge: Tennessee Williams et Michel Tremblay». TTR, 19, 1, p. 15-30.

Lane-Mercier, Gillian (1995). "La traduction des discours directs romanesques comme stratégie d'orientation des effets de lecture». Palimpsestes, 9, p. 75-92.

McKay, Ron (1996). «Would the real Irvine Welsh shoot up?» The Guardian. [http://www.theguardian.com/theobserver/1996/feb/04/ featuresreview.review] (consulté le $1^{\text {er }}$ décembre 2015).

Meschonnic, Henri (1973). Pour la poétique II: épistémologie de l'écriture poétique de la traduction. Paris, Gallimard.

Meschonnic, Henri (1982). Le poème et la voix. Lagrasse [France], Verdier.

Meschonnic, Henri (1989). La rime et la vie. Lagrasse [France], Verdier.

Meschonnic, Henri (1995a). Propos sériels: le corps et le langage. Entretiens avec Jean-Paul Desgouttes.

Meschonnic, Henri (1995b). "Traduire ce que les mots ne disent pas, mais ce qu'ils font». Meta. 40, 3, p. 514-517.

Meschonnic, Henri (2007). Éthique et politique du traduire. Lagrasse [France], Verdier.

Mitchell, James et Lynn G. Bennie (1996). «Thatcherism and the Scottish Question». In C. Rallings et al., dir. British Elections and Parties Yearbook. Londres, Éditions Frank Cass, p. 90-104.

Molière (2014 [1665]). Dom Juan, ou le Festin de pierre. Paris, Librio.

Morace, Robert (2001). Irvine Welsh's Trainspotting: A Reader's Guide. Londres, Bloomsbury Academic.

Morace, Robert (2007). Irvine Welsh. New York, Palgrave Macmillan.

Morvan, Françoise (1994). «À propos d'une expérience de traduction: Désir sous les ormes d'Eugène O'Neill». TTR, 7, 2, p. 63-92.

Ostiguy, Luc et Claude Tousignant (2008 [1993]). Les prononciations du français québécois, normes et usages. $2^{\mathrm{e}}$ éd. mise à jour. Montréal, Éditions Guérin.

Paradis, Claude et Jean Dolbec (2008). «PHONO. Applicateur de règles phonologiques». CIRAL, Université Laval. [http://linguistique. uqac.ca/recherche/phono] (consulté le $1^{\text {er }}$ décembre 2015). 
Peddie, Ian (2007). «Speaking Welsh: Irvine Welsh in Conversation». Scottish Studies Review, 8, 1, p. 130-140.

Rao, Sathya (2013). "“Traduire à l'oreille": vers une poétique de l'américanité en traduction chez Michel Tremblay». Studies in Canadian Literature/Études en littérature canadienne, 38, 2, p. 69-93.

Welsh, Irvine (1996a [1993]). Trainspotting. New York, W.W. Norton.

Welsh, Irvine (1996b). Trainspotting. Trad. Eric Lindor Fall. Paris, Éditions de l'Olivier.

Welsh, Irvine (2011). Trainspotting. Trad. Jean-René Étienne. Paris, Éditions Au diable Vauvert.

Willis, Paul. E. (1976). «The Cultural Meaning of Drug Use», in S. Hall et T. Jefferson, dir. Resistance through Rituals: Youth Subcultures in Post-war Britain. Londres, George McKay, p. 106-118.

Alex Gauthier alexgauthier.trad@gmail.com 